\title{
Surgical ablation is effective: But surgeons need to do better
}

The original "cut and sew" Cox maze procedure established an effective surgical treatment for atrial fibrillation (AF). Since then, its many iterations and the evolution of minimally invasive approaches have helped to make the surgery safer and more widely accepted. In this issue of the Journal, Henn and colleagues ${ }^{1}$ report their long-term outcomes with the "Cox maze IV procedure." Their study included 576 consecutive patients from their institution treated with either biatrial or left atrial lesion sets by sternotomy or minimally invasive approaches; most of these patients underwent concomitant cardiac procedures.

Henn and colleagues ${ }^{1}$ are strong proponents of surgical ablation to treat atrial tachyarrhythmias (ATAs), and with good reason. Patients with AF undergoing cardiac surgery have increased mortality relative to propensity-matched control subjects without AF. Lee and colleagues ${ }^{2}$ have demonstrated that successful surgical ablation eliminates this increased mortality risk and results in improved survival relative to that of unsuccessfully treated patients.

Most studies in the AF literature are retrospective analyses, as is the current report of Henn and colleagues. ${ }^{1}$ There have been, however, several randomized trials that are noteworthy. The Atrial Fibrillation Catheter Ablation versus Surgical Ablation Treatment (FAST) trial ${ }^{3}$ demonstrated superior freedom from ATAs without the need for antiarrhythmic drugs (AADs) with surgical relative to catheter ablation $(65.6 \%$ vs $36.5 \% ; P=.0022)$ in a population of patients with lone AF. A major drawback to surgical ablation was found to be a greater incidence of adverse events $(34.4 \%$ vs $15.9 \%$ with catheter ablation; $P=.027)$.

The recently published National Institutes of Health National Heart, Lung, and Blood Institute-sponsored Cardiothoracic Surgical Trials Network trial is the largest randomized, controlled AF study to date. ${ }^{4}$ Surgical ablation performed concomitantly with mitral valve surgery demonstrated superior freedom from $\mathrm{AF}$ at 12 months $(63 \%$ vs $29 \%$ with no ablation; $P<.001)$. The study was $20 \%$ of patients. by study site.

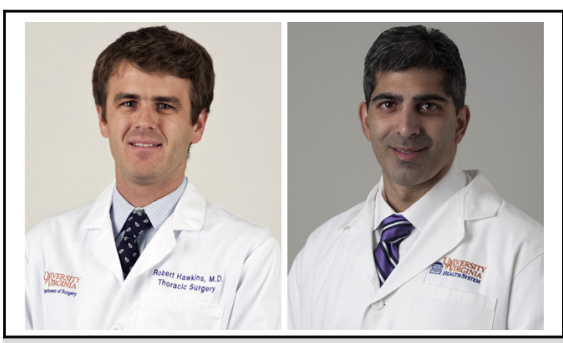

Robert B. Hawkins, MD, and Gorav Ailawadi, MD

Central Message

This article highlights long-term durability of the Cox maze procedure in expert hands and the need for multicenter prospective trials.

See Article page 1168 .

underpowered to compare the lesion sets used. Importantly, surgical ablation resulted in new pacemakers for more than

The prospective Concomitant Utilization of Radiofrequency Energy for Atrial Fibrillation (CURE-AF) trial ${ }^{5}$ had similar success in using radiofrequency ablation with a biatrial Cox maze IV procedure during concomitant surgery at 15 centers. At a follow-up of 6 to 9 months, the freedom from ATA without the need for AADs was only $53 \%$. This study reveals the impact of experience, because the heavily criticized prerequisite was only 5 cases, resulting in a freedom from ATAs ranging from $30 \%$ to $100 \%$

The report by Henn and colleagues, ${ }^{1}$ like most of the AF literature, is fraught with major inconsistencies, making interpretation of the results challenging. This includes differing types of AF, heterogeneous concomitant operations, multiple lesion sets and energy sources, and inconsistent postablation monitoring. As such, direct comparisons of surgical ablation strategies or even comparison of surgical versus catheter ablation are not appropriate. Moreover, without control patients or selection criteria, it is difficult to account for the selection bias. The only general criteria listed are for those who underwent left-sided ablation, which one could argue is not a true Cox maze IV lesion set. Finally, a main drawback to surgical ablation was its higher complication rate, which was only briefly discussed in the article.

Despite these shortcomings, there are some important findings in this report. First, the freedoms from ATAs 
without the need for AADs were respectable at 2 and 5 years at $78 \%$ and $61 \%$, respectively. These results are superior to those of the prospective trials noted here, ${ }^{4,5}$ in part because of the expertise at Washington University. Second, the addition of a box lesion improved 5-year outcomes substantially (freedom from ATAs without the need for AADs of $66 \%$ vs $33 \%$ without box lesion; $P=.017$ ). Finally, left atrial ablation alone had good results in a specific group with paroxysmal AF, left atrial size smaller than $5.0 \mathrm{~cm}$, and no right atrial enlargement (not clearly defined). A direct comparison between biatrial and left atrial lesion sets, however, could not be made because of the previously listed limitations.

There are interesting differences in risk factors for failure at 1 and 5 years. At 1 year, absence of a box lesion, left atrial size, and preoperative pacemaker were risk factors for failure. The ability to detect ATAs is likely more sensitive with an implantable device, and this may actually explain why a preoperative pacemaker appears to be a "risk factor." At 5 years, the duration of preoperative AF and length of hospital stay were predictive of failure. The surprising association of length of hospital stay was also demonstrated by Ad and coworkers. ${ }^{6}$ Although the mechanism for this association remains unclear, there are many factors that contribute to length of hospital stay, and we agree that this is likely a marker for a sicker patient at baseline or postoperatively.

Overall, Henn and colleagues ${ }^{1}$ are to be congratulated on their large experience with the Cox maze IV procedure and their excellent 5-year freedom from ATAs without the need for AADs. These results have not been reproduced in prospective trials, although these trials have included less experienced centers and more rigorous follow-up monitoring. Nevertheless, this report provides strong evidence that surgical AF ablation should be considered, especially when the preoperative duration is shorter $(<5-10$ years $)$ and left atrial size is not prohibitive. The lesion set remains an area of further investigation. Finally, this study highlights the continued need for rigorous monitoring and comparisons of homogeneous patient populations to make stronger conclusions.

\section{References}

1. Henn MC, Lancaster TS, Miller JR, Sinn LA, Schuessler RB, Moon MR, et al. Late outcomes following the Cox maze IV procedure for atrial fibrillation. J Thorac Cardiovasc Surg. 2015;150:1168-78.e2.

2. Lee R, McCarthy PM, Wang EC, Vaduganathan M, Kruse J, Malaisrie SC, et al. Midterm survival in patients treated for atrial fibrillation: a propensity-matched comparison to patients without a history of atrial fibrillation. J Thorac Cardiovasc Surg. 2012;143:1341-51; discussion 1350-1.

3. Boersma LV, Castella M, van Boven W, Berruezo A, Yilmaz A, Nadal M, et al. Atrial fibrillation catheter ablation versus surgical ablation treatment (FAST): a 2-center randomized clinical trial. Circulation. 2012;125:23-30.

4. Gillinov AM, Gelijns AC, Parides MK, DeRose JJ Jr, Moskowitz AJ, Voisine P, et al. Surgical ablation of atrial fibrillation during mitral-valve surgery. $N$ Engl J Med. 2015;372:1399-409.

5. Damiano RJ Jr, Badhwar V, Acker MA, Veeragandham RS, Kress DC, Robertson JO, et al. The CURE-AF trial: a prospective, multicenter trial of irrigated radiofrequency ablation for the treatment of persistent atrial fibrillation during concomitant cardiac surgery. Heart Rhythm. 2014;11:39-45.

6. Ad N, Holmes SD, Stone LE, Pritchard G, Henry L. Rhythm course over 5 years following surgical ablation for atrial fibrillation. Eur J Cardiothorac Surg. 2015; 47:52-8; discussion 58 . 